\title{
Standard Model physics at ATLAS
}

\author{
E. Yu. Soldatov ${ }^{1, *}$ \\ on behalf of the ATLAS Collaboration** \\ ${ }^{1}$ National Research Nuclear University «Moscow Engineering Physics Institute», 115409, Kashirskoe \\ shosse, 31, Moscow, Russia
}

\begin{abstract}
The latest advances in Standard Model measurements performed by the ATLAS experiment at the LHC are discussed. Vector-boson scattering processes have been observed in several final states $(Z Z, W Z$, same-sign $W W)$, and evidence has been reached in the $Z \gamma$ final state. The experimental precision of QCD measurements has reached the same order as the theoretical uncertainties on the NNLO calculations. Evidence for the triboson $W V V$ final state has been achieved. The latest results on $V+$ jets, $Z \gamma, W^{+} W^{-}$and direct photons production are presented. New results on jet substructure and forward proton tagging program are also discussed.
\end{abstract}

\section{Introduction}

The Standard Model (SM) of particle physics has been successful at explaining and predicting all results obtained at collider experiments (and others) so far. Expectations of this theory have been confirmed many times with a high level of precision. SM measurements in collider experiments are used for its verification on the one hand and disproof or extension on the other hand.

To reach the first goal, we aim to validate the SM in a new energy regime and improve the precision of known SM parameters. To reach the second goal, we probe for beyond Standard Model (BSM) contributions (e.g. anomalous couplings, etc) and provide the information about the SM processes, which are backgrounds to direct BSM searches.

This article reports the SM results from the ATLAS Collaboration that are available less than a year after the end of LHC Run 2. At this moment, ATLAS has published almost 200 SM-related papers [2]. This overview will cover just a few recent ones on different topics. The covered topics are: Electroweak Physics (single $W$ and $Z$ bosons, Dibosons/Tribosons, vector-boson fusion/scattering production), Direct photons, Jet Physics, Soft QCD, Diffraction and Forward Physics.

\section{Data taking by ATLAS}

Thanks to the excellent performance of the LHC during Run 1, the ATLAS detector collected $4.6 \mathrm{fb}^{-1}$ of $7 \mathrm{TeV}$ collision data and $20.3 \mathrm{fb}^{-1}$ of $8 \mathrm{TeV}$ collision data. During Run 2, the ATLAS detector collected $139 \mathrm{fb}^{-1}$ of $13 \mathrm{TeV}$ collision data, which are ready to be analysed.

\footnotetext{
*e-mail: EYSoldatov@mephi.ru

${ }^{* *}$ Copyright 2019 CERN for the benefit of the ATLAS Collaboration. CC-BY-4.0 license.
} 
The pile-up conditions in Run 2 were worse than in Run 1 and the average number of interactions in one bunch crossing exceeded 40 for 2017 and 2018 data taking. Also, some low pile-up runs were taken, for precise $Z$ and $W$ bosons measurements.

In Figure 1 (left), a schematic illustration of the ATLAS detector [1] and its components, is shown. Besides the well-known "central" parts, ATLAS also includes forward detectors used for important elastic proton scattering measurements (e.g. ALFA, shown on the right in Figure 1).
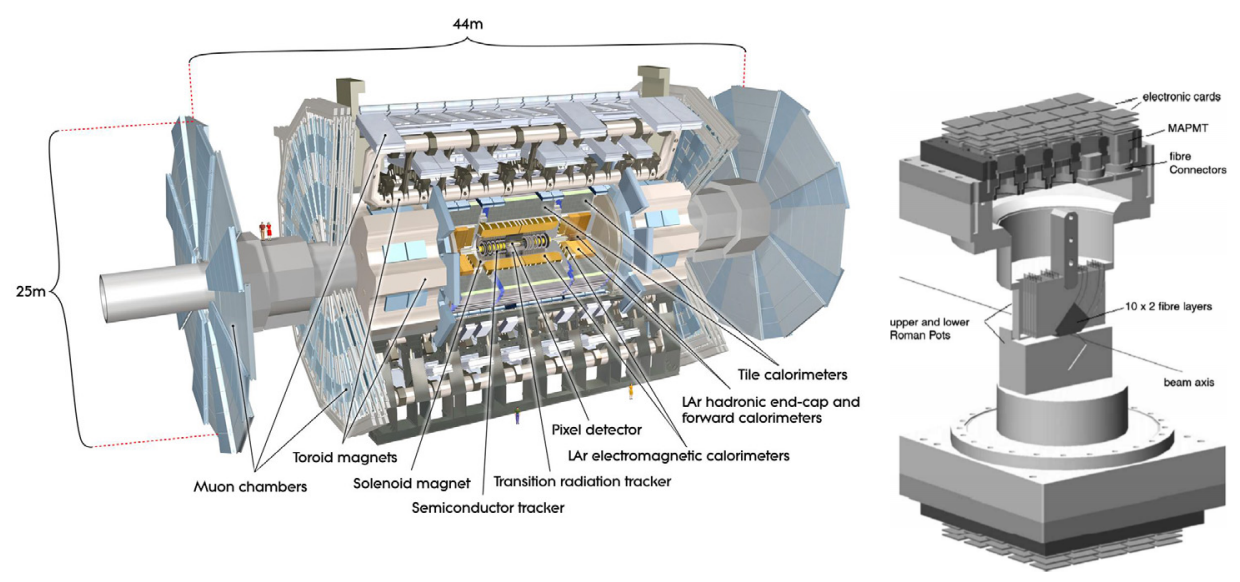

Figure 1. Left: ATLAS detector scheme. Right: scheme of upper and lower parts of the ALFA detector and their Roman Pots.

\section{Electroweak measurements}

\subsection{Single boson production}

Single electroweak boson production is a benchmark process for fixed-order calculations and predictions of MC simulations for perturbative QCD ( $\mathrm{pQCD}$ ). The high precision reached in these measurements can be used for the study of parton density functions (PDFs).

The measurement of inclusive single $Z$ boson production, with the $Z$ decaying to an electron-positron pair, has been performed using $19.9 \mathrm{fb}^{-1}$ of $8 \mathrm{TeV}$ data [3]. The main backgrounds for this study come from the multijet and $W+$ jet processes. The theoretical NNLO QCD predictions with different PDF sets, obtained using the NNLOJET [5] framework, are in good agreement with the measured cross sections, within uncertainties, as shown in Figure 2. NLO predictions have worse agreement with the data. The dominant uncertainties for the measurement are from the signal modelling and the systematic uncertainties related to jet energy resolution and scale.

Other inclusive measurements of $W$ and $Z$ single production have been performed under special conditions with low pile-up and using $4 \mathrm{pb}^{-1}$ of data with a centre-of-mass collision energy of $2.76 \mathrm{TeV}$ [4]. The main backgrounds come from electroweak (diboson, single boson and $\tau$ decays) and multijet processes. The theoretical NNLO predictions with different PDF sets are in good agreement with the measured cross sections within uncertainties, 

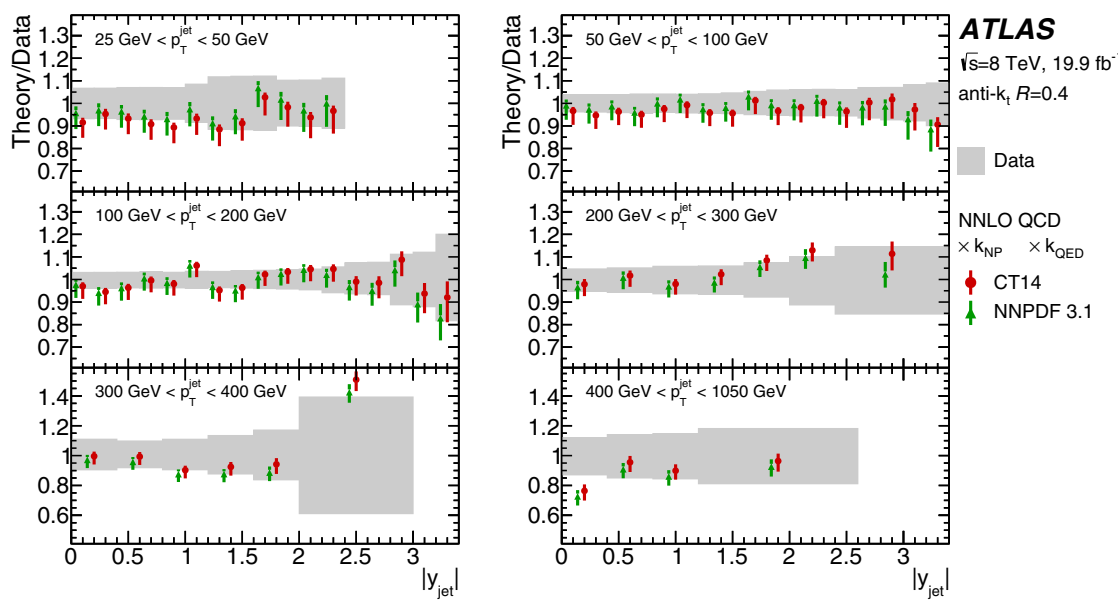

Figure 2. Ratio of the measured $Z+$ jets production cross section and the NNLO QCD predictions, obtained using NNLOJET, corrected for the non-perturbative and QED radiation effects as a function of $\left|y_{\text {jet }}\right|$ and $p_{\mathrm{T}}^{\text {jet }}$ bins. The theoretical predictions are calculated using various PDF sets. The coloured error bars represent the sum in quadrature of the effects of the PDF, scale, and $\alpha_{S}$ uncertainties, and the uncertainties from the non-perturbative and QED radiation corrections. The grey band shows the sum in quadrature of the statistical and systematic uncertainties in the measurement except for the luminosity uncertainty of $1.9 \%[3]$.

as shown in Figure 3. The dominant uncertainties for the measurement are from limited statistics, lepton reconstruction and identification efficiency simulation, and from imperfect knowledge of the multijet background. Using the cross-section ratios for $W^{+} / W^{-}$and $W / Z$, different PDF sets have been tested, as shown in Figure 4. The main idea of this study relies upon the fact that some of the uncertainties will cancel out in ratios and the ratio will be more sensitive to the PDFs. A slight tension between the data and the prediction using the ABMP16 PDF set is observed.
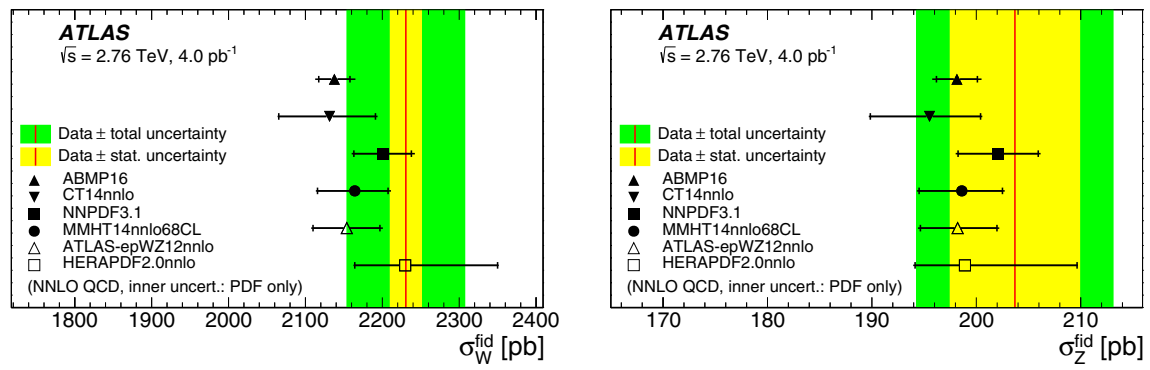

Figure 3. NNLO predictions for the fiducial cross sections $\sigma_{W}^{\text {fid }}$ (left) and $\sigma_{Z}^{\text {fid }}$ (right) for the six PDFs: CT14nnlo [6], MMHT2014 [7], NNPDF3.1 [8], ATLASepWZ12 [9], ABMP16 [10] and HERApdf2.0 [11] compared with the measured fiducial cross section. The inner shaded band represents the statistical uncertainty only, the outer band corresponds to the experimental uncertainty (including the luminosity uncertainty). The theory predictions are given with the corresponding PDF (total) uncertainty shown by the inner (outer) error bar [4]. 

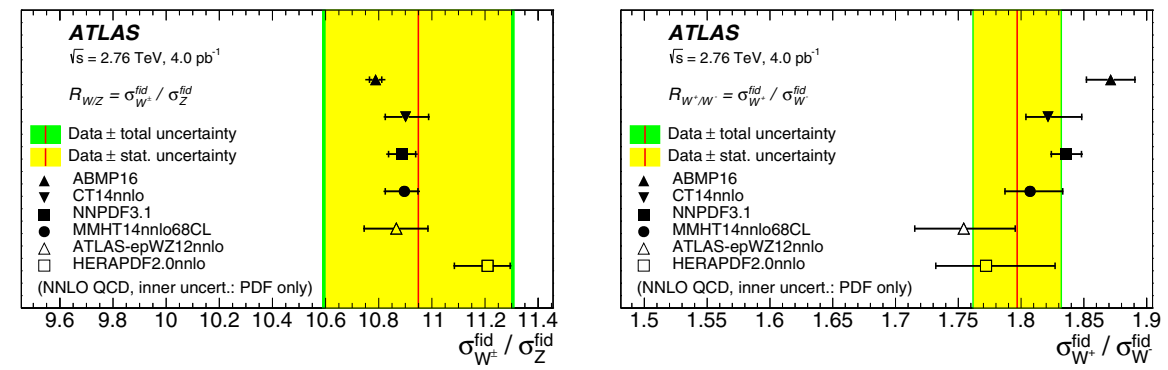

Figure 4. The measured ratio of fiducial cross sections for $W$ boson production to $Z$ boson production (left), $W^{+}$boson production to $W^{-}$boson production (right). The measurements are compared with theoretical predictions at NNLO in QCD based on a selection of different PDF sets. The inner shaded band corresponds to the statistical uncertainty while the outer band shows statistical and systematic uncertainties added in quadrature. The theory predictions are given with the corresponding PDF (total) uncertainty shown by the inner (outer) error bar [4].

\subsection{Vector-boson fusion/scattering production}

Vector-boson fusion/scattering $(\mathrm{VBF} / \mathrm{VBS})$ processes are the rarest SM processes currently accessible to the LHC experiments. The cross sections of these processes are on the level of a few femtobarns or even lower. These processes are an extremely sensitive tool to test the SM predictions and search for anomalous boson couplings. Also, these processes represent the irreducible backgrounds for VBF Higgs production.

For these analyses, ATLAS is using several new common techniques:

- Due to difficult simulation of dominant backgrounds (especially its normalization), background enriched control regions are used to constrain them;

- Extraction of the cross section is performed by simultaneous fit instead of simple counting;

- Machine learning techniques, like boosted decision trees (BDT), are used in order to separate the electroweak (EWK) and strong (QCD) components (with QCD vertices in diagrams) of the studied final states;

- The effective field theory approach is used to parametrize the anomalous couplings.

First, the measurement of electroweak $Z Z$ production using the full Run 2 dataset of $139 \mathrm{fb}^{-1}$ [12] with $\sqrt{s}=13 \mathrm{TeV}$ is discussed. The 4-lepton and the 2-lepton+2-neutrino modes were considered. The theoretical NLO SM prediction is in a good agreement with the measured integrated cross section of QCD+EWK components of the $Z Z$ production, as shown in Figure 5. To obtain the EWK cross section, the fitting of a BDT output distribution in the signal region (SR) was used. The measured cross section is equal to $0.82 \mathrm{fb}$, and the observed signal strength is about $1.35 \pm 0.34$. The observed significance is $5.5 \sigma$, which constitutes the first observation of the EWK ZZ process. The dominant uncertainties for the measurement are from limited statistics, luminosity, and systematic uncertainties from the leptons and jets momentum scale and resolution.

The next measurement presented here is the electroweak $Z \gamma$ production, based on $36.1 \mathrm{fb}^{-1}$ of $13 \mathrm{TeV}$ data [13]. Only electron and muon decays of the $Z$ were considered. The theoretical SM prediction, obtained with the MG5_aMC@NLO [14] and Sherpa [15] $\mathrm{MC}$ generators, is in agreement with the measured integrated cross section of QCD+EWK components of the $Z \gamma$ production: 

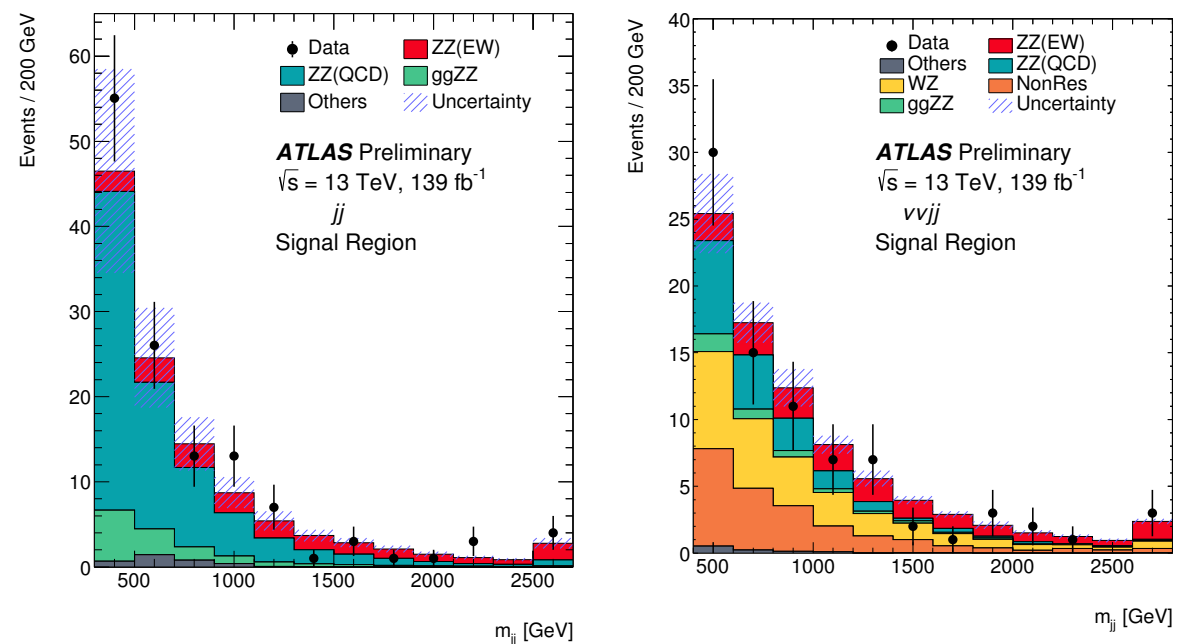

Figure 5. Observed and expected $m_{\mathrm{jj}}$ distributions in the $\ell \ell \ell \ell j j$ (left) and $\ell \ell v v j j$ (right) signal regions. The error bands include the expected experimental and theoretical uncertainties. The error bars on the data points show the statistical uncertainty on data. The contributions from the QCD and EWK production of $Z Z j j$ events are scaled by 0.96 and 1.35 , respectively, which correspond to the observed normalization factors in the statistical fit to the combined channel. The last bins include the overflow events [12].

Measurement: $\sigma_{Z \gamma j j}^{\text {fid. }}=71 \pm 2$ (stat.) ${ }_{-7}^{+9}$ (exp.syst.) ${ }_{-17}^{+21}$ (mod.syst) fb;

Prediction: $\sigma_{Z \gamma j j}^{\text {fid.Madgraph+Sherpa }}=88.4 \pm 2.4$ (stat.) $\pm 2.3\left(\mathrm{PDF}+\alpha_{S}\right)_{-19.1}^{+29.4}$ (scale) fb.

Again, to obtain the EWK-only cross section, the fitting of the BDT output distribution in the SR was performed. The theoretical LO prediction is in good agreement with the measured signal cross section:

Measurement: $\sigma_{Z \gamma j j}^{\text {fid. }}=7.8_{-1.4}^{+1.5}$ (stat. ${ }_{-1.0}^{+0.9}$ (syst. ${ }_{-0.8}^{+1.0}$ (mod.) fb;

Prediction: $\sigma_{Z \gamma j j}^{\text {fid.Madgraph }}=7.75 \pm 0.03$ (stat.) $\pm 0.20\left(\mathrm{PDF}+\alpha_{S}\right) \pm 0.40$ (scale) fb.

The observed significance is $4.1 \sigma$, which constitutes evidence from ATLAS of the EWK $Z \gamma$ process. The dominant uncertainties for the measurement are from limited statistics, jet energy scale and from the heavy flavor tagging efficiency.

For the measurement of electroweak $W Z$ production based on $36.1 \mathrm{fb}^{-1}$ of $13 \mathrm{TeV}$ data [16], only leptonic decay modes of the bosons were considered. The theoretical prediction obtained with Sherpa is in agreement with the measured integrated cross section of QCD+EWK components of $W Z$ production:

Measurement: $\sigma_{W^{ \pm} Z j j}^{\text {fid. }}=1.68 \pm 0.16$ (stat.) \pm 0.12 (exp.syst.) \pm 0.13 (mod.syst.) \pm 0.44 (lumi.) fb;

Prediction: $\sigma_{W^{ \pm} Z j j}^{\text {fid.Sherpa }}=2.15 \pm 0.01$ (stat.) \pm 0.05 (PDF) ${ }_{-0.44}^{+0.65}$ (scale) fb.

After the fitting of the BDT output distribution in the SR, the MC LO prediction, obtained with Sherpa, is in fair agreement with the measured signal EWK-only cross section:

Measurement: $\quad \sigma_{W^{ \pm} Z j j}^{\text {fid. }}=0.57{ }_{-0.13}^{+0.14}$ (stat.) ${ }_{-0.04}^{+0.05}$ (exp.syst.) ${ }_{-0.04}^{+0.05}$ (mod.syst.) ${ }_{-0.01}^{+0.01}$ (lumi.) fb;

Prediction: $\sigma_{W^{ \pm} Z j j}^{\text {fid.Sherpa }}=0.321 \pm 0.002$ (stat.) \pm 0.005 (PDF) ${ }_{-0.023}^{+0.027}$ (scale) fb. 
The observed significance is $5.3 \sigma$, which constitutes the first observation of the EWK $W Z$ process. Differential cross sections of QCD+EWK components were measured as a function of several observables, one of them is shown in Figure 6. The dominant uncertainties for the measurement are from limited statistics, and systematic uncertainties from the jet energy scale and MC modelling.
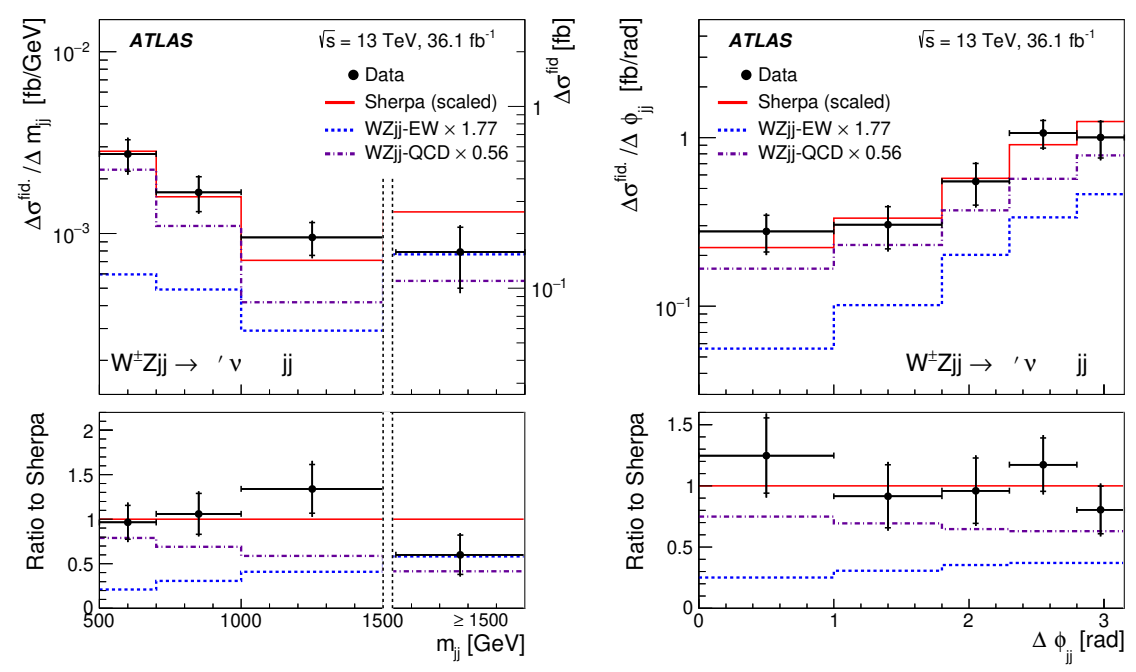

Figure 6. The measured $W^{ \pm} Z j j$ differential cross section in the VBS fiducial phase space as a function of the invariant mass of the tagged jets $m_{\mathrm{jj}}$ (left) and the azimuthal angle between the two tagged jets $\Delta \phi_{\mathrm{jj}}$ (right). The inner and outer error bars on the data points represent the statistical and total uncertainties, respectively. The measurements are compared with the sum of the rescaled $W Z \mathrm{jj}-\mathrm{QCD}$ and $W Z \mathrm{jj}-\mathrm{EWK}$ predictions from Sherpa (solid line). The $W Z \mathrm{jj}-\mathrm{EWK}$ and $W Z \mathrm{jj}-\mathrm{QCD}$ contributions are also represented by dashed and dashed-dotted lines, respectively. In (left), the right $y$-axis refers to the last cross-section point, separated from the others by a vertical dashed line, as this last bin is integrated up to the maximum value reached in the phase space. The lower panels show the ratios of the data to the predictions from Sherpa [16].

The measurement of the electroweak same-sign $W W$ production is also based on $36.1 \mathrm{fb}^{-1}$ of $13 \mathrm{TeV}$ data [17]. Only leptonic decay modes of the $W$ boson were considered. To obtain the EWK cross section, $m_{\mathrm{jj}}$ distribution in the SR was fitted. The observed signal strength is $1.44 \pm 0.38$, and the observed significance is $6.5 \sigma$, which constitutes an observation by ATLAS of the EWK same-sign $W W$ process. The dominant uncertainties for the measurement are from limited statistics, and systematic uncertainties from MC modelling and the estimation of the background from misidentified leptons.

In summary, it should be noted that such rare processes are accessible for measurement only at the LHC experiments, and most of them have been observed within the last 5 years. At the time of writing, only $Z \gamma$ and $W \gamma$ processes are yet to be observed.

\subsection{Diboson and triboson production}

Multiboson production is an important high precision test of the SM. In addition, diboson processes are the main backgrounds for VBS and Higgs measurements. 
The measurement of $Z \gamma$ production based on the full Run 2 dataset has used only electron and muon decays of the $Z$ boson [18]. The goal of this study is the measurement of differential cross sections (as a function of $E_{\mathrm{T}}^{\gamma}, \eta^{\gamma}, m_{l l \gamma}$ and $p_{\mathrm{T}}^{l l \gamma}$ ). The MATRIX [19] NNLO theoretical predictions are in a good agreement with the data, as shown in Figure 7. The dominant uncertainties for the measurement are from limited statistics, and systematic uncertainties related to the $Z+$ jets background estimation and the photon efficiency.
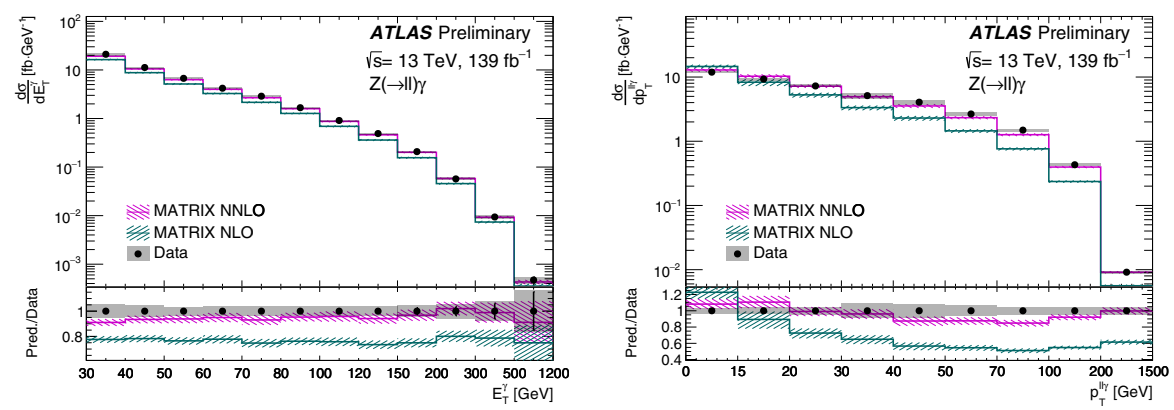

Figure 7. Measured fiducial cross sections (black data points) for the observables $E_{\mathrm{T}}^{\gamma}$ (left) and $p_{\mathrm{T}}(\ell \ell \gamma)$ (right) for the $p p \rightarrow Z\left(\rightarrow \ell^{+} \ell^{-}\right) \gamma$ process. The measured cross sections are compared with SM expectations obtained from the MATRIX parton-level generator, corrected to particle level. The error bars on the data points show the statistical uncertainty on the measured values. The grey shaded regions show the total uncertainty on the unfolded data, excluding the uncertainty on the integrated luminosity. The purple and green hatched regions show the total uncertainty on the MATRIX predictions. The lower section of each plot shows the ratio of the SM expectation to the measured cross section [18].

In this analysis, there is a possibility that a $Z$ and a photon are produced, originating from different primary vertices, which leads to an additional pile-up background. This background was found to contribute $5 \%$ to the total event yield.

Another $Z \gamma$ production measurement with the $Z$ boson decaying into neutrinos was performed with $36.1 \mathrm{fb}^{-1}$ of $13 \mathrm{TeV}$ data [20]. The theoretical NNLO MCFM [21] predictions are in good agreement with the measured integrated cross sections in inclusive and exclusive fiducial volumes:

Measurement (inclusive): $\sigma_{Z(v v) \gamma}^{\text {ext.fid. }}=83.7_{-3.5}^{+3.6}$ (stat.) ${ }_{-6.2}^{+6.9}$ (syst.) ${ }_{-2.0}^{+1.7}$ (lumi.) fb;

Prediction (inclusive): $\sigma_{Z(v v) \gamma}^{\text {ext.fid.MCFM }}=78.1 \pm 0.2$ (stat.) \pm 4.7 (syst.) fb.

The measurement of differential cross sections were performed as a function of $p_{\mathrm{T}}^{\gamma}$, jet multiplicity and $E_{\mathrm{T}}^{\text {miss }}$, which are in agreement with the NNLO MCFM predictions, as shown in Figure 8.

Limits on anomalous neutral triple gauge couplings ( $Z Z \gamma$ and $Z \gamma \gamma)$ were set, as shown in Table 1. The obtained limits are the best ones on neutral aTGCs at the time of writing.

A further measurement is that of $W W$ production at $13 \mathrm{TeV}$ [22]. Integrated and differential cross sections were measured, which are in agreement with the MATRIX NNLO prediction, as shown in Figure 9. Limits on the anomalous couplings were also set, as shown in Table 2.

Finally, the latest triboson analysis of $W V V$ production [25] is presented. The final states considered in the measurement are: $W W W \rightarrow \ell v \ell v q q, W W W \rightarrow \ell v \ell v \ell v, W W Z \rightarrow \ell v q q \ell \ell$, 

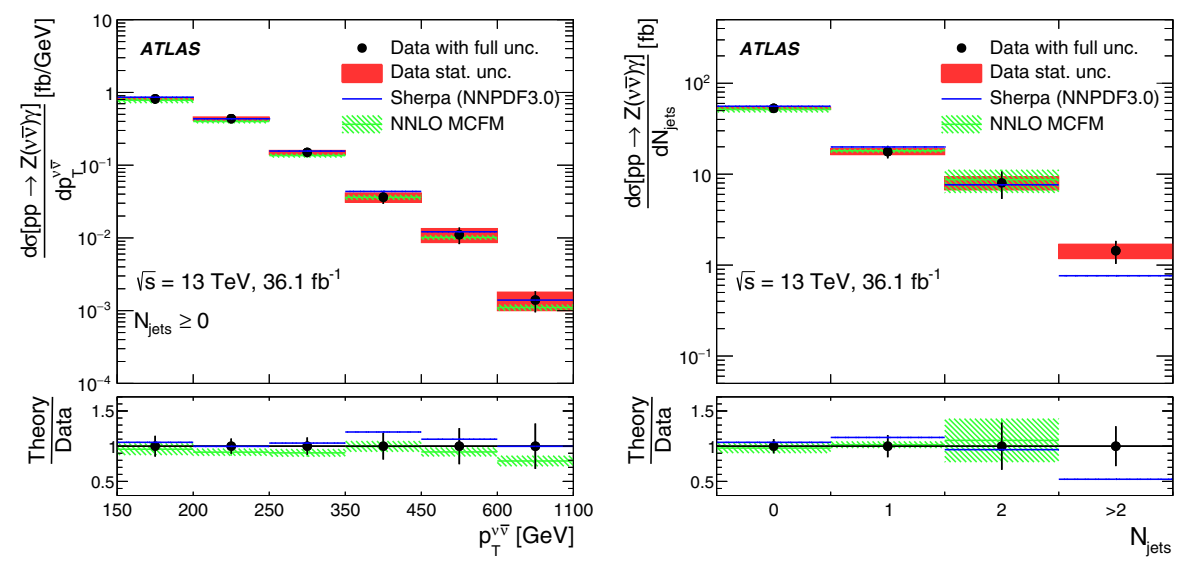

Figure 8. The measured (points with error bars) and predicted differential cross sections as a function of $p_{\mathrm{T}}^{v v}$ (left) and $N_{\text {jets }}$ (right) for the $p p \rightarrow Z(v v) \gamma$ process. The error bars on the data points show the sum in quadrature of the statistical and systematic uncertainties. The MCFM NNLO predictions are shown with shaded bands that indicate the theoretical uncertainties. The lower plots show the ratios of the SM expectation to the measured values (shaded bands), with the error bars on the points showing the relative uncertainties in the experimental measurements [20].

Table 1. Observed and expected one-dimensional 95\% CL limits on the $C_{\tilde{B} W} / \Lambda^{4}, C_{B W} / \Lambda^{4}, C_{W W} / \Lambda^{4}$ and $C_{B B} / \Lambda^{4}$ EFT parameters, assuming that any excess in data over the SM expectation is due solely to a non-zero value of the parameter $C_{\tilde{B} W} / \Lambda^{4}, C_{B W} / \Lambda^{4}, C_{W W} / \Lambda^{4}$ or $C_{B B} / \Lambda^{4}$. For each row, all parameters other than the one under study are set to $0[20]$.

\begin{tabular}{lll}
\hline Parameter & \multicolumn{2}{c}{ Limit $95 \% \mathrm{CL}$} \\
& Measured $\left[\mathrm{TeV}^{-4}\right]$ & Expected $\left[\mathrm{TeV}^{-4}\right]$ \\
\hline$C_{\tilde{B} W} / \Lambda^{4}$ & $(-1.1,1.1)$ & $(-1.3,1.3)$ \\
$C_{B W} / \Lambda^{4}$ & $(-0.65,0.64)$ & $(-0.74,0.74)$ \\
$C_{W W} / \Lambda^{4}$ & $(-2.3,2.3)$ & $(-2.7,2.7)$ \\
$C_{B B} / \Lambda^{4}$ & $(-0.24,0.24)$ & $(-0.28,0.27)$ \\
\hline
\end{tabular}

Table 2. The expected and observed 95\% CL intervals for the anomalous coupling parameters of the EFT model [22].

\begin{tabular}{lll}
\hline Parameter & \multicolumn{2}{c}{ Limit 95\% CL } \\
& Measured $\left[\mathrm{TeV}^{-2}\right]$ & Expected $\left[\mathrm{TeV}^{-2}\right]$ \\
\hline$C_{W W W} / \Lambda^{2}$ & $(-3.4,3.3)$ & $(-3.0,3.0)$ \\
$C_{W} / \Lambda^{2}$ & $(-7.4,4.1)$ & $(-6.4,5.1)$ \\
$C_{B} / \Lambda^{2}$ & $(-21,18)$ & $(-18,17)$ \\
$C_{\tilde{W} W W} / \Lambda^{2}$ & $(-1.6,1.6)$ & $(-1.5,1.5)$ \\
$C_{\tilde{W}} / \Lambda^{2}$ & $(-76,76)$ & $(-91,91)$ \\
\hline
\end{tabular}

$W W Z \rightarrow \ell v \ell v \ell \ell, W Z Z \rightarrow q q \ell \ell \ell \ell$, (where $\ell=e / \mu$ ). The main backgrounds for this measurement come from $W Z+$ jets and processes containing non-prompt leptons. The measurement of the integrated cross section was performed for all the final states. The prediction is in agreement with the measured cross sections, as shown in Figure 10. The combination of studied final states gives an observed significance of $4.1 \sigma$, providing evidence for this process. 

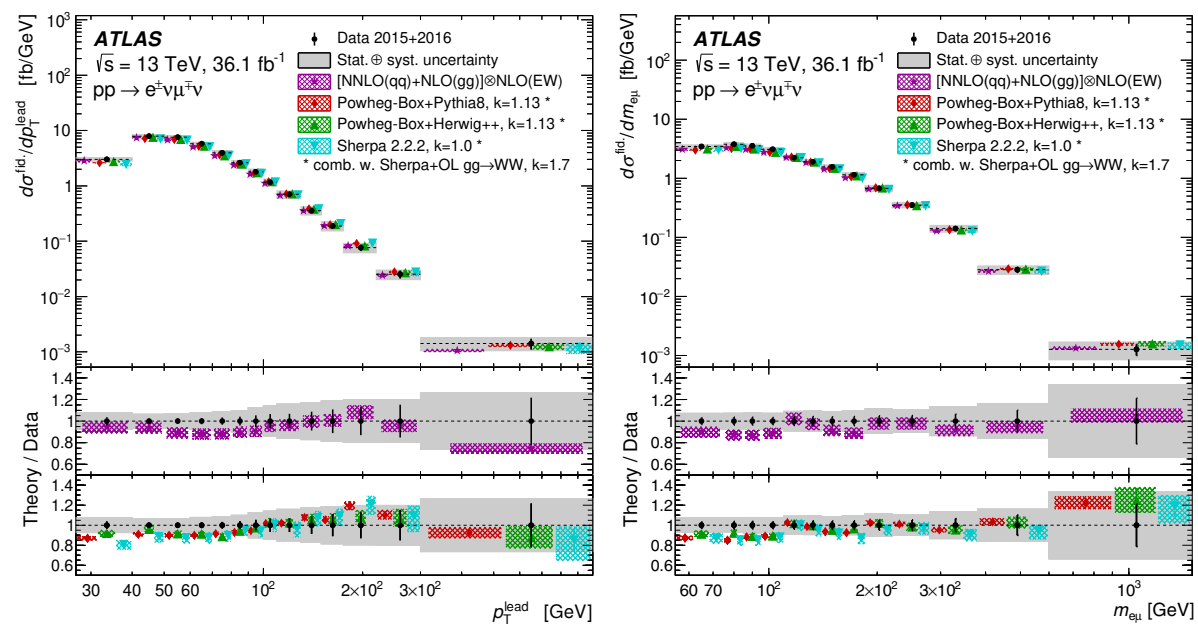

Figure 9. Measured fiducial cross sections of $W W \rightarrow e \mu$ production $p_{\mathrm{T}}^{\text {lead } \ell}$ (left) and $m_{e \mu}$ (right). The measured cross section values are shown as points with error bars giving the statistical uncertainty and solid bands indicating the size of the total uncertainty. The results are compared with the NNLO prediction with extra NLO EWK corrections and NLO corrections for $g g \rightarrow W W$ production, and with NLO+PS predictions from Powheg-Box+Pythia 8 [23], Powheg-Box+Herwig++ [24] and Sherpa 2.2.2 for $q \bar{q}$ initial states, combined with Sherpa+Openloops (LO+PS) for the $g g$ initial states. All three $q \bar{q}$ NLO+PS predictions are normalized to the NNLO theoretical prediction for the total cross section, with the $g g \mathrm{LO}+\mathrm{PS}$ contribution normalized to NLO. The theoretical predictions are indicated as markers with hatched bands denoting PDF+scale uncertainties [22].

\section{Direct photon production}

The study of direct photons is a test of perturbative QCD with a hard colourless probe. Also, it is a testground for different MC models of prompt-photon production.

The ATLAS Collaboration has performed measurements of differential cross-section ratios for direct photons at different collision energies [26]. The first ratio, $R_{13 / 8}^{\gamma}$, shown on the left of Figure 11, is the ratio of the photon cross section measured at $\sqrt{s}=13 \mathrm{TeV}$ to that at $8 \mathrm{TeV}$. The second, $D_{13 / 8}^{\gamma / Z}$, is shown on the right of Figure 11, and is the double ratio given by $R_{13 / 8}^{\gamma}$ divided by the analogous ratio for $Z$ boson production. The theoretical predictions are in agreement with the data in most of the bins. Since some systematic uncertainties cancel in the ratios, the photon energy scale error becomes comparable to other systematic contributions.

A further photon analysis is the cross-section measurement at $13 \mathrm{TeV}$, based on $36.1 \mathrm{fb}^{-1}$ of data [27]. The differential cross sections for inclusive photon production were measured. The main background for photons comes from the misidentification of jets, so the selection of photons was designed to suppress this contamination. ATLAS provides photon isolation and electromagnetic calorimeter shower shape identification requirements to suppress such misidentification. The NNLO predictions are in very good agreement with the measured cross sections, as shown in Figure 12. The dominant systematic uncertainty on the measurement is due to the photon energy scale. 


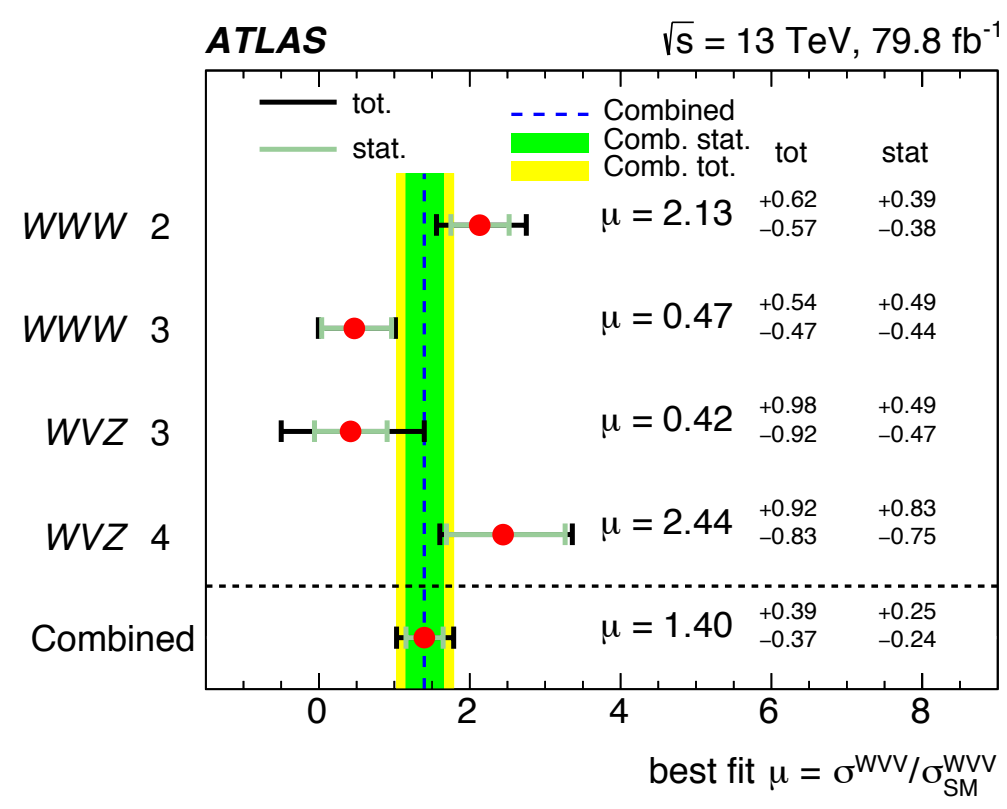

Figure 10. Extracted signal strengths $\mu$ for the four analysis regions of $W V V$ production and for the combination [25].

\section{Jet physics}

Study of hadronic jets provides an advanced way to test QCD. Jet measurements are sensitive to the strong coupling and proton PDFs, and it is possible to explore the evolution of high energy quarks and gluons into hadrons. It can be also used to understand the origin of jets from the light quarks, gluons or hadronic decays of some heavy particles.

The first measurement discussed here is dedicated to studies of jet internal substructure [29]. Using $\sqrt{s}=13 \mathrm{TeV}$ data, measurements of several jet substructure observables for trimmed and soft drop jets, were performed. The full list of variables and the details about each can be found in the published paper [29]. Differential cross sections were measured and compared to MC generator predictions. As shown in Figure 13, the data is best described by Madgraph5+Pythia8, though none of the generator predictions is able to completely model the data.

ATLAS has also produced a "Lund plane" measurement [30], which provides information on the internal structure and formation of jets. The main idea is that the jet may be approximated as soft emissions around a hard core, which represents the originating quark or gluon. So the Lund plane is defined as: $\ln (1 / z)$ versus $\ln (1 / \theta)$, where $z$ is the relative momentum of the emission with respect to the jet core and $\theta$ is the opening angle of emission to the jet core. The measurement of the differential cross section of the Lund plane was performed, and is shown in Figure 14.

The Lund plane naturally factorizes perturbative and non-perturbative effects, the underlying event, etc. One of the most important applications of the Lund plane is the possibility to use it in the machine-learning-based discriminants. 

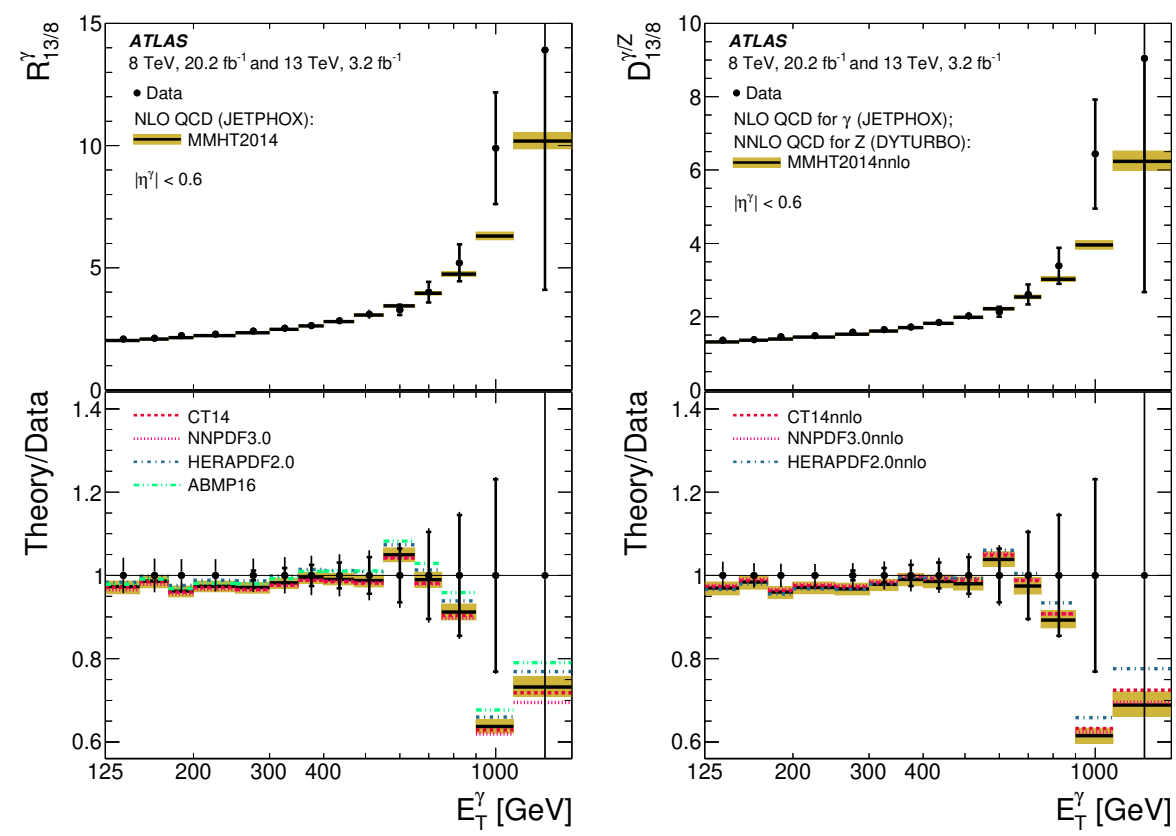

Figure 11. The measured $R_{13 / 8}^{\gamma}$ (left) and $D_{13 / 8}^{\gamma / Z}$ (right) as a function of $E_{\mathrm{T}}^{\gamma}$ in $\left|\eta^{\gamma}\right|<0.6$ region. The NLO QCD predictions based on the MMHT2014 PDFs (black lines) are also shown. The inner (outer) error bars represent the statistical (total) uncertainties. The shaded band represents the theoretical uncertainty in the predictions. The lower part of the figures shows the ratio of the NLO QCD predictions based on the MMHT2014 PDFs to the measured $R_{13 / 8}^{\gamma}$ or $D_{13 / 8}^{\gamma / Z}$ (black lines) [26].

\section{Forward physics}

As described in section 2, ATLAS has forward detectors such as ALFA. The latest measurement from the forward program is the measurement of single diffractive dissociation at $\sqrt{s}=8 \mathrm{TeV}[31]$.

The study of soft QCD is well motivated. Since most of the diffraction kinematics is characterized by the soft scales, it can not be described by pQCD, and it is therefore an important tool to probe the strong interaction in the non-perturbative regime. Phenomenological pomeron models are used to describe the interactions in the diffraction studies. The special LHC runs with high $\beta^{*}$, which corresponds to the weaker focusing of the hadron beams, are performed for diffraction physics measurements.

The result of the study is the measurement of differential cross sections as a function of a number of variables that characterize diffractive dissociation: the squared four-momentum transfer of the proton $(t)$; the momentum fraction of the proton carried by the pomeron $(\xi)$; and the (pseudo)rapidity gap from the edge of the tracker $(\Delta \eta)$.

The results are shown in Figure 15. For the rapidity gap (shown on the left), the MC models give a reasonable description of the shape, though do not describe the overall crosssection normalization. For the other observables, an exponential model was used and the slope measured, as shown on the right of Figure 15. The exponential slope parameter value, predicted by Pythia8 with the A2 tune to be $7.82 \mathrm{GeV}^{-2}$, is compatible with the measurement, $B=7.60 \pm 0.32 \mathrm{GeV}^{-2}$. 


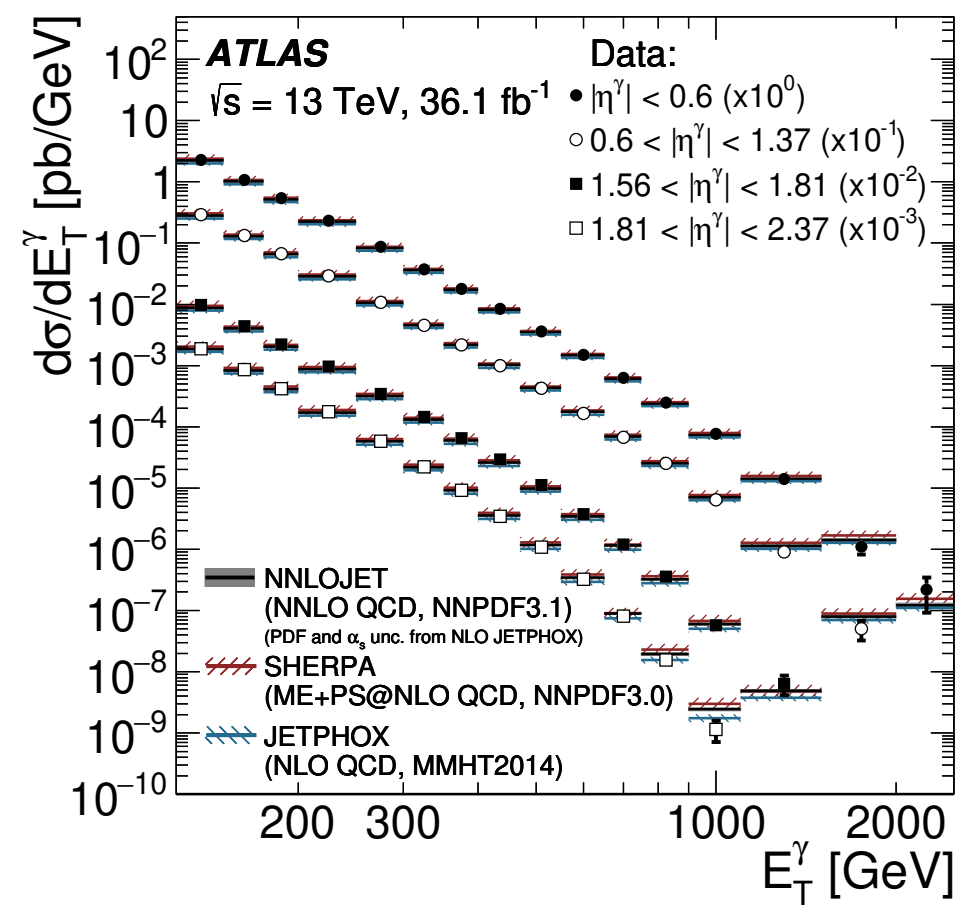

Figure 12. The measured differential cross section for isolated-photon production as a function of $E_{\mathrm{T}}^{\gamma}$ in $\left|\eta^{\gamma}\right|<0.6$ (black dots), $0.6<\left|\eta^{\gamma}\right|<1.37$ (open circles), $1.56<\left|\eta^{\gamma}\right|<1.81$ (black squares) and $1.81<\left|\eta^{\gamma}\right|<2.37$ (open squares). The NLO pQCD prediction from JETPHOX [28], the ME+PS@ NLO QCD prediction from Sherpa 2.2.2 and the NNLO QCD prediction from NNLOJET are also shown. The measurement and the predictions are normalised by the factors shown in parentheses to aid visibility. The error bars represent the data statistical uncertainties and systematic uncertainties added in quadrature. For most of the points, the error bars are smaller than the marker size and, thus, not visible. The bands represent the theoretical uncertainty associated with the predictions; in the case of NNLOJET, the uncertainties due to the PDFs and $\alpha_{s}$ are estimated at NLO with JETPHOX [27].

\section{Conclusions}

Tests of the Standard Model, using the ATLAS experiment at the LHC, include the measurement of cross sections over 14 orders of magnitude, as shown in Figure 16, ranging from inclusive proton-proton and jets cross sections, through to the rare triboson and VBS processes.

Early ATLAS full Run 2 analyses and precision Run 1 analyses provide very stringent tests of SM.

New SM measurements covering a variety of topics have been presented. Electroweak measurements give results which are consistent with the SM, and several rare SM processes have been observed. Photon measurements demonstrate good agreement with NNLO QCD predictions, and can be used to provide the input for improving MC photon production models. Measurements of the jet shape observables and the Lund plane are promising for taggers. Diffractive proton-proton cross sections have been measured for the first time, and are found 

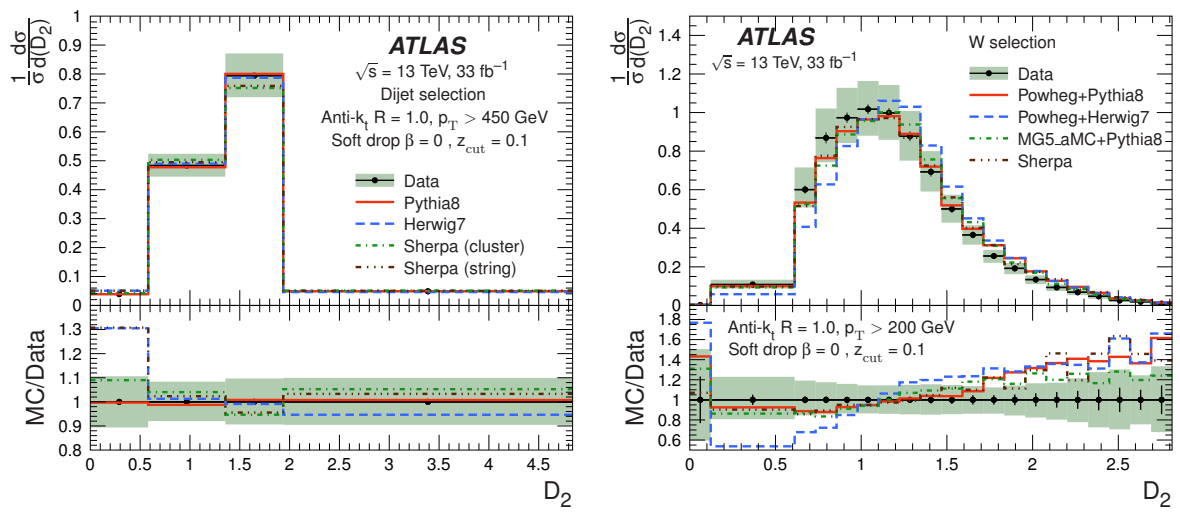

Figure 13. The distributions of $D_{2}$ compared with different MC predictions for soft-dropped largeradius jets from dijet (left) and $W$ (right) selections. For the dijet selection, Sherpa is tested with two different hadronisation models. Data are compared between the soft-dropped large-radius jets for the selections mentioned above. The shaded bands represent the total uncertainty, while the error bars show the statistical uncertainty [29].

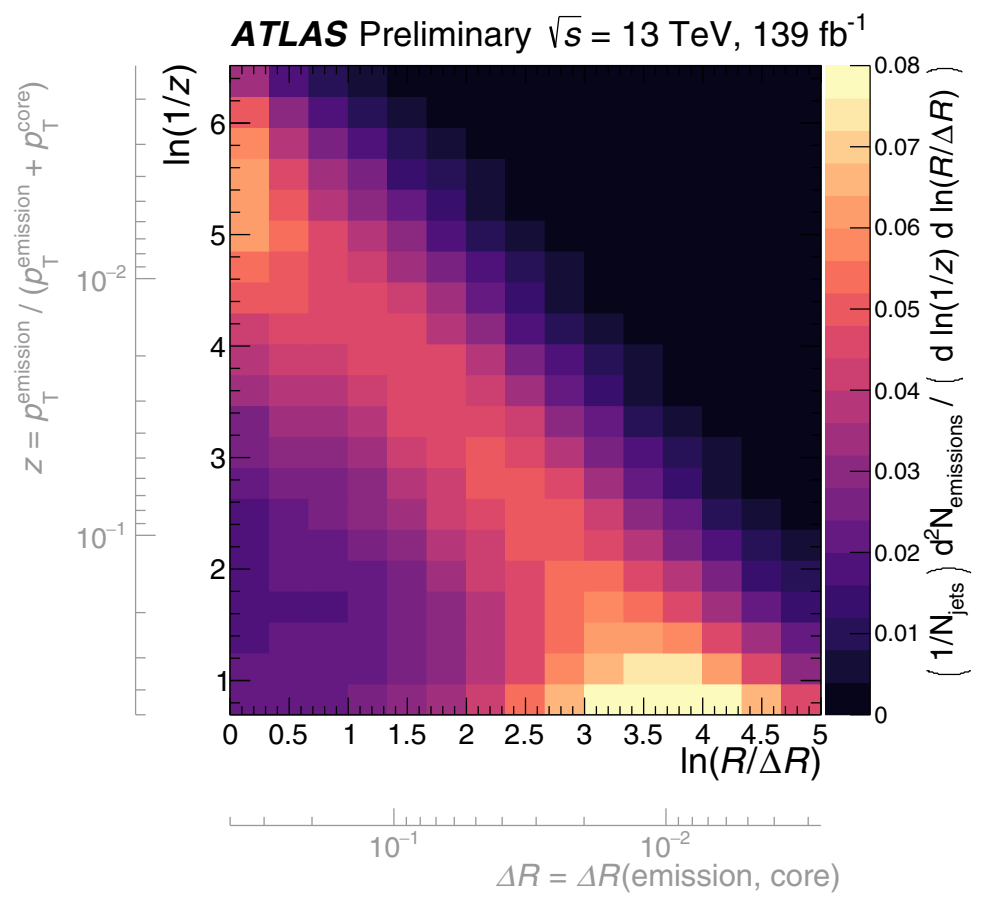

Figure 14. The Lund jet plane as measured using jets in $13 \mathrm{TeV} p p$ collision data, corrected to particlelevel. The inner set of axes indicate the coordinates of the Lund jet plane itself, while the outer set indicate corresponding values of $z$ and $\Delta R[30]$. 

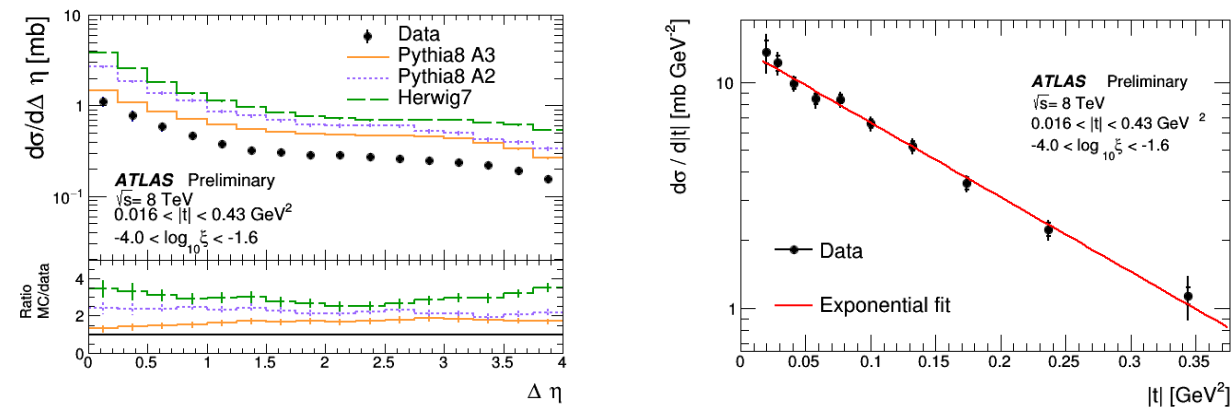

Figure 15. Left: Hadron level differential cross section as a function of $\Delta R$, comparing the measured data with Pythia8 and Herwig7 predictions. The error bars display the combination of statistical and systematic uncertainties in quadrature. Right: The differential cross section as a function of $|t|$ with inner error bars representing statistical uncertainties and outer error bars displaying the statistical and systematic uncertainties added in quadrature [31].

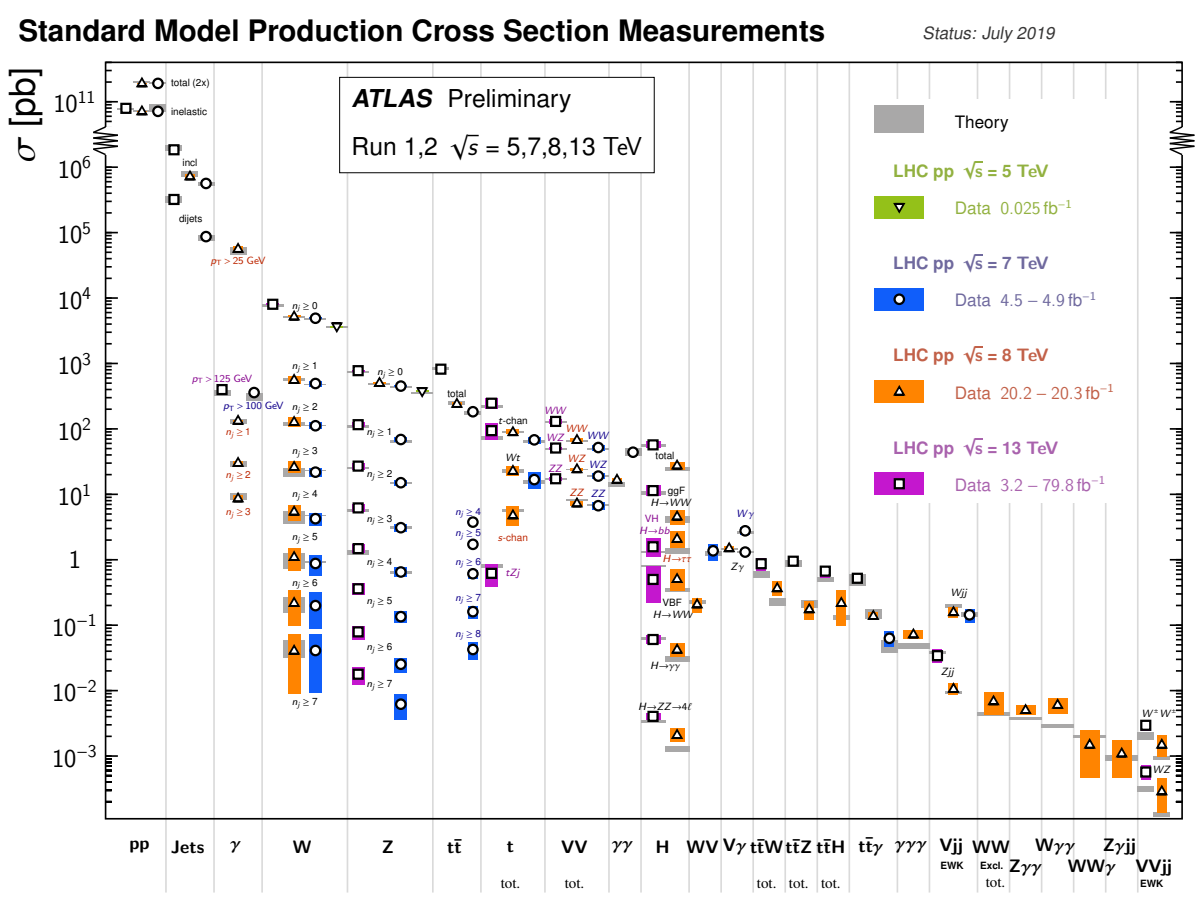

Figure 16. Summary of several Standard Model total and fiducial production cross section measurements, corrected for branching fractions, compared to the corresponding theoretical expectations [2].

to be in agreement with the theoretical models. Deviations from SM expectations, such as anomalous couplings, have not been observed, and strong limits have been obtained.

Many different analyses based on the full Run 2 dataset are still in progress and the results of new, strong tests of the SM will become available soon. 


\section{Acknowledgements}

The reported study was funded by RFBR according to the research project N 18-32-20160.

\section{References}

[1] ATLAS Collaboration, JINST 3, S08003 (2008)

[2] https://twiki.cern.ch/twiki/bin/view/AtlasPublic/StandardModelPublicResults

[3] ATLAS Collaboration, https://arxiv.org/abs/1907.06728

[4] ATLAS Collaboration, https://arxiv.org/abs/1907.03567

[5] R. Gauld et al., JHEP 1711, 003 (2017)

[6] S. Dulat et al., Phys. Rev. D 93, 033006 (2016)

[7] L. A. Harland-Lang et al., Eur. Phys. J. C 75, 204 (2015)

[8] R. D. Ball et al., Eur. Phys. J. C 77, 663 (2017)

[9] ATLAS Collaboration, Phys. Rev. Lett. 109, 012001 (2012)

[10] S. Alekhin et al., Phys. Rev. D 96, 014011 (2017)

[11] H1 and ZEUS Collaborations, Eur. Phys. J. C 75, 580 (2015)

[12] ATLAS Collaboration, ATLAS-CONF-2019-033, http://cdsweb.cern.ch/record/2682845

[13] ATLAS Collaboration, https://arxiv.org/abs/1910.09503

[14] J. Alwall et al., JHEP 1407, 158 (2014)

[15] T. Gleisberg et al., JHEP 0902, 007 (2009)

[16] ATLAS Collaboration, Phys. Lett. B 793, 469 (2019)

[17] ATLAS Collaboration, Phys. Rev. Lett. 123, 161801 (2019)

[18] ATLAS Collaboration, ATLAS-CONF-2019-034, http://cdsweb.cern.ch/record/2682846

[19] M. Grazzini et al., Eur. Phys. J. C 78, 537 (2018)

[20] ATLAS Collaboration, JHEP 1812, 010 (2018)

[21] J. M. Campbell et al., JHEP 1107, 018 (2011)

[22] ATLAS Collaboration, https://arxiv.org/abs/1905.04242

[23] T. Sjöstrand et al., Comput. Phys. Commun. 191, 159 (2015)

[24] M. Bahr et al., Eur. Phys. J. C 58, 639 (2008)

[25] ATLAS Collaboration, Phys. Lett. B 798, 134913 (2019)

[26] ATLAS Collaboration, JHEP 1804, 093 (2019)

[27] ATLAS Collaboration, Phys. Lett. B 780, 578 (2018)

[28] S. Catani et al., JHEP 0205, 028 (2002)

[29] ATLAS Collaboration, JHEP 1908, 033 (2019)

[30] ATLAS Collaboration, ATLAS-CONF-2019-035, http://cdsweb.cern.ch/record/2683993

[31] ATLAS Collaboration, ATLAS-CONF-2019-012, http://cdsweb.cern.ch/record/2670978 